\title{
MINIMAL SURFACE MAPS, FIXED POINT INDICES AND A JIANG-GUO TYPE INEQUALITY
}

\author{
MICHAEL R. KELLY \\ Department of Mathematics and Computer Science \\ Loyola University, New Orleans, LA 70118, U.S.A. \\ E-mail: kelly@beta.loyno.edu
}

1. Introduction. One approach to getting information about the dynamics of a continuous mapping $f: X \rightarrow X$ is to describe a minimal dynamical representative $\phi$ for the homotopy class to which $f$ belongs. This representative should have two inherent features. First, it should have the least amount of "dynamics" among all maps in the homotopy class, and secondly, the dynamical information should be computable.

Neither of these conditions are absolutes. So in context one must decide as to what "least dynamics" means and what type of information one would like to compute. For example, asymptotic information such as the topological entropy of the map, or local information such as the index of a fixed point. The book $[\mathrm{KH}]$ is a good reference for dynamical terms and results mentioned in this paper. The book $[\mathrm{Br}]$ gives a thorough treatment of the fixed point index and its relation to the topology of the space $X$.

Perhaps a good choice for the lest amount of dynamics is that $\phi$ have the least number of periodic points possible for all periods. That is, the least number of fixed points among all maps in the homotopy class, the least number of period two points, and so on. As such a map may not exist, an alternative might be to relax the conditions imposed on the map at the expense of uniqueness of representative and ease of computation.

A simple example that illustrates the idea of a minimal dynamical representative is when $X$ is (homeomorphic to) the unit sphere in 2-dimensional Euclidean space. Let $\mathbb{R}$ denote the real line as the universal cover of $X$. Then the map $\phi_{k}: \mathbb{R} \rightarrow \mathbb{R}$ defined by $\phi_{k}(x)=k x$ projects to an expanding map on $X$ which has the least dynamics among maps of degree $k$. These minimal maps have the feature that any two fixed points have the same index; -1 when $k \geq 2$, +1 when $k \leq 0$. More generally, linear maps defined on the $n$-torus $S^{1} \times \cdots \times S^{1}$ act as minimal dynamical representatives. If all of the

1991 Mathematics Subject Classification: Primary 55M20; Secondary 58C30.

Key words and phrases: fixed point index, minimal map, surface.

The paper is in final form and no version of it will be published elsewhere. 
eigenvalues for the matrix associated to this map have modulus different from one, then the fixed points are isolated and they all have the same index, $I= \pm 1$. In this setting the number of fixed points of a map $f$ is easily computed using the formula

$$
N F(f)=|L(f)|,
$$

where $L(f)$ denotes the Lefschetz number of the mapping, and $N F(f)$ the number of fixed points of $f$. Generalizations of this idea can be found in the work of a number of authors. See $[\mathrm{M}]$ for a survey of some of these results.

A more interesting and useful setting for the notion of a minimal representative occurs in dimension two. When $X$ is a compact surface, and $h: X \rightarrow X$ is a homeomorphism there is a natural choice for a minimal dynamical representative as a result of the work of Thurston [T], [CB]. Using an elaborate limiting construction he defined certain homeomorphisms which are known by the name pseudo-Anosov. Although pseudo-Anosov homeomorphisms were difficult to explicitly construct, a variety of their dynamical properties were established. See, for example, [FLP], [HP] or [Bo].

Among the numerous known results about pseudo-Anosov's the following one serves as motivation for the work of this paper. A consequence of the classical Euler-Poincaré formula for singular foliations of surfaces (see [FLP; Exposé 5]) is that for each fixed point $x$ of of the pseudo-Anosov homeomorphism $h$,

$$
2 \chi(X)-1 \leq \operatorname{index}(x) \leq 1,
$$

where $\chi(X)$ denotes the Euler characteristic of $X$.

Now, not every surface homeomorphism is homotopic to a pseudo-Anosov. By using Thurston's classification theorem [T], Jiang and Guo [JG] define a class of representative homeomorphisms that have the feature that any given surface homeomorphism is isotopic to one of them. In addition, each member has exactly the number of fixed points predicted by the Nielsen number, and the inequality stated above holds as well. See $[\mathrm{Br}]$ or $[\mathrm{J}]$ for the definition and properties of the Nielsen number.

In $[\mathrm{JG}]$ the authors use the estimates on the indices of fixed points to establish the inequality

$$
|L(h)-\chi(X)| \leq N(h)-\chi(X),
$$

where $N(h)$ denotes the Nielsen number of $h$. The authors raise the question as to whether or not this same inequality holds for all self-mappings of surfaces. For surfaces with nonempty boundary, Wagner [W] gives evidence for an affirmative answer to this question by showing that the inequality holds for a certain technical class of self-mappings.

When $f: X \rightarrow X$ is a continuous self-mapping of a compact surface which is not homotopic to a homeomorphism there is no parallel notion to that of pseudo-Anosov. Nor is it clear that there is a minimal representative which realizes the periodic point data for all periods. Our approach in this paper is to consider two classes of maps that will play the role of minimal representatives: fixed-point minimal maps and geometrically minimal maps. The fixed-point minimal class is dynamically minimal in a very weak sense, while the geometrically minimal maps are simple topologically but not based on any dynamical information. 
The purpose of this paper is to generalize the inequalities stated above for homeomorphisms of surfaces to both of these classes of self-mappings of surfaces. These are stated as Theorems 1.1-1.4 below. The definitions of fixed-point minimal and geometrically minimal are postponed until the next section.

Let $F$ be a compact connected surface with non-empty boundary. Its Euler characteristic is denoted by $\chi(F)$.

THEOREM 1.1. If $f: F \rightarrow F$ is a geometrically minimal self-mapping, then for each fixed point $x$ of $f$,

$$
2 \chi(F)-1 \leq \operatorname{index}(x) \leq 1 .
$$

THEOREM 1.2. If $f: F \rightarrow F$ is a fixed-point minimal self-mapping, then for each fixed point $x$ of $f$,

$$
2 \chi(F)-1 \leq \operatorname{index}(x) \leq 1 .
$$

The inequality of Jiang and Guo stated earlier can be viewed as the Lefschetz number providing a lower bound for the Nielsen number of a surface homeomorphism. Since the Nielsen number is a lower bound for the number of fixed points, a weaker form is that the inequality gives a lower bound for the number of fixed points. For a self-mapping $f: F \rightarrow F$ let $N F(f)$ denote the cardinality of the set of fixed points of $f$. In [K3] this weak version of the Jiang-Guo inequality is established. Namely,

THEOREM 1.3. Let $F$ be a surface with non-empty boundary. Then for any selfmapping $f: F \rightarrow F$,

$$
|L(f)-\chi(F)| \leq N F(f)-\chi(F) .
$$

Note that Theorem 1.3 is really a theorem about minimal maps. If the inequality holds for minimal maps, then clearly it holds for all maps. In this paper we prove the weaker analog for geometrically minimal maps.

THEOREM 1.4. Let $F$ be a surface with nonempty boundary and let $f: F \rightarrow F$ be a geometrically minimal self-mapping. Then

$$
|L(f)-\chi(F)| \leq N F(f)-\chi(F) .
$$

The proofs of Theorems 1.2 and 1.3 are quite technical and will appear in [K3]. In Section 3 of this paper we give the proofs of the results for geometrically minimal maps. These proofs are much simpler and turn out to contain the motivating ideas behind the results for fixed-point minimal maps. In fact, the relationship between the class of geometrically minimal maps and the class of fixed-point minimal maps has been studied by the author. Some of this work appears in [K2]. The method of proof relies quite heavily on the fact that the surface has non-empty boundary. The corresponding results for closed surfaces is unknown at this time.

2. Minimal maps on surfaces. Let $F$ be a compact connected surface with nonempty boundary. It is well known that such surfaces have a handle decomposition with no 2-handles. We view $F$ as being equipped with a handle structure consisting of a single disk $D$, the 0 -handle, together with $k$-handles attached to $D$, where $k=1-\chi(F)$. Let $A$ denote the union of the $2 k$ attaching arcs for the 1 -handles. 
Definition. A map $f: F \rightarrow F$ is said to be geometrically minimal if there exists a handle structure (as described above) such that each component of $f^{-1}(A)$ is either an essential simple closed curve or a proper arc in $F$, and has minimal geometric intersection with $A$. Furthermore, we assume that no fixed points occur on $A$, and that each component of $F \backslash\left(f^{-1}(A) \cup A\right)$ contains at most one fixed point.

REMARKS. Here minimal intersection means up to isotopy, with boundary going to boundary. Note that in the special case that $F$ is a disk, $A=\emptyset$ and minimality just means that $f$ has one fixed point. Also, the last condition in the definition may appear to be restrictive. It is not however, as the other conditions imply that each component of $F \backslash\left(f^{-1}(A) \cup A\right)$ is a disk. As a result, a map defined on the frontier of a component extends to the interior with at most one fixed point.

We state the following without proof. The result is not used in this paper.

Proposition 2.1. Each homotopy class of self-maps of F contains a geometrically minimal map.

Definition. A map $f: F \rightarrow F$ is said to be fixed-point minimal if $N F(f)$ is the least number of fixed points possible among all maps homotopic to $f$. Equivalently, $f$ is fixed-point minimal when it has exactly $M F[f]$ fixed points.

Here are two examples related to the results in this paper. The first illustrates that the lower bounds given in Theorems 1.1 and 1.2 are sharp. For self-mappings of surfaces $M F[f]$ is generally not easy to compute. The paper [K1] was devoted to this computation in the very special case of the pants surface. Example 2 gives a situation where Theorem 1.2 can be used to compute $M F[f]$.

EXAMPLE 1. Let $F$ be a surface with non-empty boundary. Let $W$ denote a wedge of $n$ circles $\omega_{1}, \ldots, \omega_{n}$ embedded in $F$ and based at a point $p$. We assume that $n$ and $W$ are chosen so that the inclusion $i: W \rightarrow F$ is a homotopy equivalence. Define a map $f: W \rightarrow W$ by $\omega_{i} \mapsto \omega_{i} \omega_{i}$ for each $i$, and such that $\operatorname{Fix}(f)=p$. Extend to a map $f: F \rightarrow W$. Define $g: F \rightarrow F$ by $g(x)=i(f(x))$. Clearly, $p$ is the only fixed point of $g$ so $g$ is fixed-point minimal. Since $L(g)=2 \chi(F)-1$, it follows that index $(p)=2 \chi(F)-1$.

REMARK. The map can also be defined so that it is geometrically minimal as well. In fact, after choosing a handle structure one can arrange that $g^{-1}(A) \cap A=\emptyset$.

ExAmple 2. Let $F$ and $W$ be as in Example 1. Let $f: F \rightarrow F$ be a map and express the induced map on the fundamental group in the form $\omega_{i} \mapsto W_{i}$ where for each $i, W_{i}$ is a reduced word. Suppose that $\omega_{i}$ does not appear in $W_{i}$, and let $m_{i}$ be the number of appearences of $\omega_{i}^{-1}$ in $W_{i}$. Then $M F[f]=L(f)=1+\sum m_{i}$. The reason is simply that $f$ is homotopic to a map (with image in $W$ ) having exactly $1+\sum m_{i}$ fixed points, each with index +1 . Thus, any map with fewer fixed points would have one with index strictly greater than one.

Let $C$ be a component of $F \backslash\left(f^{-1}(A) \cup A\right)$ and let $\partial C$ denote its frontier. Let $s(C)$ denote the number of components of $\partial C \cap f^{-1}(A)$, and let $p(C)$ denote the number of 
points of $A \cap \partial F$ in $C$. Set $q(C)=s(C)+\frac{p(C)}{2}$. With this notation we have the following combinatorial analog to the Euler-Poincaré formula.

Proposition 2.2. $\sum_{C}(2-q(C))=2 \chi(F)$.

Proof. Embed a graph $G$ in the 0-handle $D$ so that each component $C$ contains exactly one vertex, and two vertices are joined by an edge whenever the corresponding components have frontier in common. Note that $G$, which is dual to the collection of curves $f^{-1}(A) \cap D$, is connected and acyclic. Let $e$ be the number of edges of $G$. Then there are $e+1$ vertices in $G$. By definition, $\sum_{C} s(C)=2 e$, where the sum is taken over all regions in $D$. Since $f$ is geometrically minimal, $\sum_{C} p(C)$ is just the cardinality of $A \cap \partial F$, which is equal to $4 k$ or $4(1-\chi(F))$. Thus, summing again in $D$,

$$
\sum_{X}(2-q(C))=2(e+1)-\left(2 e+\frac{1}{2}(4(1-\chi(F)))\right)=2 \chi(F) .
$$

Finally, for geometrically minimal maps, we have that $q(C)=2$ for each $C$ not contained in $D$, which completes the proof.

3. Proofs of Theorems 1.1 and 1.4. As in Section $2, C$ will denote a component of $F \backslash\left(f^{-1}(A) \cup A\right)$. The index of $C$ is the topological fixed point index of $f$ on the open disk $C$ (see $[\mathrm{Br}]$ or $[\mathrm{J}])$.

Proposition 3.1. Let $C$ be a component of $F \backslash\left(f^{-1}(A) \cup A\right)$ such that $f(C)$ and $C$ are contained in the same handle. Then $1-s(C) \leq \operatorname{index}(C) \leq 1$.

Remark. The hypothesis that $f(C)$ and $C$ belong to the same handle is minor. If not, then $\operatorname{index}(C)=0$. On the other hand, when $C$ is an entire handle $s(C)=0$ and the left hand inequality fails.

Proof. Let $E$ denote the handle containing $C$ and let $\zeta$ be a subarc of $E \cap \partial F$ that misses $C$. (If no such $\zeta$ exists, minimality implies that $X=E$ and the result follows.) Define a homeomorphism $h: E \rightarrow[0,1] \times[0,1]$ such that $h(\zeta)=(\{1\} \times[0,1]) \cup$ $([0,1] \times\{0,1\})$. Fix an orientation on $E$, and give $\partial C$ the induced orientation. The homeomorphism $h$ induces an orientation on $[0,1] \times[0,1] \subset \mathbb{R}^{2}$ and hence, on the unit sphere $S^{1}$ in $\mathbb{R}^{2}$. Define the unit vector field $\phi: \partial C \rightarrow S^{1}$ on $\partial C$ by

$$
\phi(x)=\frac{h(x)-h(f(x))}{|h(x)-h(f(x))|} .
$$

Since $\partial C$ is a simple closed curve the degree of $\phi$ is well defined and is equal to index $(C)$.

The regular value $(0,1) \in S^{1}$ will be used to compute the degree of $\phi$. This is computed by considering the action of $\phi$ on a neighborhood of each point in $\phi^{-1}((0,1))$. First observe that $\phi^{-1}((0, \pm 1))$ consists of all of the endpoints of the components of $\partial C \cap f^{-1}(A)$. Let $\theta$ denote the unique component that separates $\zeta$ from $C$ in $E$. For a component $\beta$ other than $\theta, \phi^{-1}((0,1))$ contains either none, one or both of its endpoints. If both occur, then locally $\phi$ reverses orientation at one and preserves orientation at the other. Thus, there is no contribution to the degree. The feature of geometrically minimal used here is that if exactly one endpoint belongs to $\phi^{-1}((0,1))$, then $\beta$ separates $C$ from $f(\beta)$ in $E$ and thus, $\phi$ is locally orientation reversing at this point. This contributes -1 to the degree. 
For the component $\theta$ the local action of $\phi$ is the opposite, resulting in a contribution of either 0 or 1 to the degree. The result now follows.

Proof of Theorem 1.1. Choose a handle structure that satisfies the definition of geometrically minimal. Let $x$ be a fixed point of $f$ and let $C$ be the component of $F \backslash\left(f^{-1}(A) \cup A\right)$ that contains $x$. From the definition, $\operatorname{index}(x)=\operatorname{index}(C)$. The result now follows from Proposition 3.1 as $s(C) \leq 2 \chi(F)$ always.

Proof of Theorem 1.4. Proving the inequality in Theorem 1.4 is equivalent to showing that $L(f) \leq N F(f)$ and $L(f)+N F(f) \geq 2 \chi(F)$.

Let $\mathcal{C}$ denote the set consisting of those components of $F \backslash\left(f^{-1}(A) \cup A\right)$ which have a non-zero index. By Proposition 3.1, for each $C \in \mathcal{C}$, index $(C) \leq 1$. Sum over $\mathcal{C}$ to get

$$
\sum_{\mathcal{C}} \operatorname{index}(C) \leq \sum_{\mathcal{C}} 1
$$

The left hand side of this inequality contains all of the fixed point index for $f$ while the right hand side counts the number of fixed points. Thus,

$$
L(f) \leq N F(f) .
$$

For each $C \in \mathcal{C}$, Proposition 3.1 also implies that

$$
\text { index }(C)+1 \geq 2-q(C) \text {. }
$$

Sum over $\mathcal{C}$ to get

$$
L(f)+N F(f) \geq \sum(2-q(C)) .
$$

Now, for each region not in $\mathcal{C}$ it follows that $0 \geq(2-q(C))$. Sum over all these regions and combine with the inequality above to get the same inequality, but now the right hand sum is over all components of $F \backslash\left(f^{-1}(A) \cup A\right)$. By Proposition 2.2, this is just $2 \chi(F)$ and so we have established

$$
L(f)+N F(f) \geq 2 \chi(F) .
$$

\section{References}

[Bo] P. Boyland, Topological methods in surface dynamics, Topology Appl. 58 (1994), 223-298.

[Br] R. F. Brown, The Lefschetz Fixed Point Theorem, Scott-Foresman, Chicago, 1971.

[CB] A. CAsson and S. BleILER, Automorphisms of Surfaces after Nielsen and Thurston, London Math. Soc. Stud. Texts 9, Cambridge University Press, Cambridge, 1988.

[FLP] A. Fathi, F. Laudenbach and V. PoÉnaru, Travaux de Thurston sur les surfaces, Astérisque 66-67 (1979).

[HP] J. Harer and R. Penner, The Combinatorics of Train Tracks, Ann. of Math. Stud. 125, Princeton Univ. Press, 1992.

[J] B. JiAng, Lectures on Nielsen Fixed Point Theory, Contemp. Math. 14, Amer. Math. Soc., Providence, RI, 1983.

[JG] B. JiAng and J. GuO, Fixed points of surface diffeomorphisms, Pacific J. Math. 160 (1993), 67-89. 
[KH] A. Katok and B. Hasselblatt, Introduction to the Modern Theory of Dynamical Systems, Encyclopedia Math. Appl. 54, Cambridge Univ. Press, Cambridge, 1995.

[K1] M. R. KELLY, Minimizing the number of fixed points for self-maps of compact surfaces, Pacific J. Math. 126 (1987), 81-123.

[K2] M. R. KELLY, Minimizing the cardinality of the fixed point set for selfmaps of surfaces with boundary, Michigan Math. J. 39 (1992), 201-217.

[K3] M. R. Kelly, A bound on the fixed point index for surface mappings, to appear in Ergodic Theory Dynam. Systems.

[M] C. K. McCord, Computing Nielsen numbers, in: Contemp. Math. 152, Amer. Math. Soc., 1993, 249-267.

[T] W. P. Thurston, On the geometry and dynamics of diffeomorphisms of surfaces, Bull. Amer. Math. Soc. 19 (1988), 417-431.

[W] J. WAGNER, An algorithm for calculating the Nielsen number on surfaces with boundary, preprint, UCLA, 1995. 